\title{
Welcome to the Special Section on Advanced Image Technology
}

\author{
Editor-in-Chief Kazuhito Murakami
}

It is a great pleasure to introduce the special section "Advanced Image Technology" in the Transactions on Media Technology and Applications (MTA). Since the inauguration of the MTA, the editorial committees have been offering a special section in each issue. This is the 4th topic in series of these special sections focusing on the advances in image, video, and multimedia technologies in a wide-spread of areas ranging from fundamental studies to applications. Initially, we called for papers in the areas of

- Image / video processing

- Image / video coding

- 3DTV / FTV (Free-viewpoint Television)

- Pattern recognition

- Computer graphics

- Computer vision

- Image / video quality assessment
- Broadcasting / communication

- ITS (Intelligen Transportation Systems)

- Multimedia applications / systems

- Virtual reality

- Animation

- Data hiding

- Other related works of Advanced Image Technology.

With the great effort made by the associate editors in the guest editorial committee and anonymous reviewers, we have selected eight high quality papers.

We are very grateful to all the authors who have submitted their excellent work to this issue and all the guest editors and reviewers. We hope this special section will promote reseach in each field.

\section{MTA Editorial Committee members list (Vol.1, No.4, 2013)}

\section{Editor-in-Chief}

Kazuhito Murakami

\section{Secretary}

Ken Tsutsuguchi

\section{Associate Editors}

Yoshimitsu Aoki

Shogo Muramatsu

Mamoru Doke

Takahiro Ogawa

Hideyuki Ebine

Masahiro Okuda

Miki Haseyama

Mie Sato

Kota Hidaka

Jun Shimamur

Toshihiko Hiroaki

Asako Soga

Hiroyasu Negishi
Aichi Prefectural University

NTT Media Intelligence Lab.

$\begin{array}{ll}\text { Masahiro Tada } & \text { Kinki University } \\ \text { Yasunobu Hitomi } & \text { SONY } \\ \text { Norio Tagawa } & \text { Tokyo Metropolitan University } \\ \text { Atsuro Ichigaya } & \text { NHK } \\ \text { Mehrdad P. Tehrani } & \text { NICT } \\ \text { Koichi Ito } & \text { Tohoku University } \\ \text { Tohru Tsuruta } & \text { FUJITSU } \\ \text { Shinichiro Koto } & \text { TOSHIBA } \\ \text { Osamu Watanabe } & \text { Takushoku University } \\ \text { Kenji Matsuo } & \text { KDDI R\&D Laboratories } \\ \text { Mahito Fujii } & \text { NHK } \\ \text { Nobuyuki Yagi } & \text { Tokyo City University }\end{array}$

